\title{
Benign Mucous Membrane Pemphigoid: A Case Report
}

\section{NIDHI OJHA', OM PRAKASH YADAV*2 (D)}

Benign Mucous Membrane Pemphigoid (MMP) is a vesiculobullous (VB) lesion that affects oral and nasal mucosa, oropharynx conjunctiva, and genital mucosa. It is considered an autoimmune disease. Autoantibodies are produced due to several external factors or genetic factors. It creates many complications, such as defects in vision, voice alteration (hoarseness), airway obstruction, and dyspareunia. The subepithelial blisters form due to these antibodies. Treatment modalities differ from topical steroids to systemic steroid therapy based on the nature and severity of clinical symptoms.

KEYWORDS: Autoimmune Disease, Pemphigoid, Mucous Membrane

\section{INTRODUCTION}

Vesiculobullous (VB) diseases are a group of oral disorders denoted by the development of vesicles or bullae. VB group of diseases comprise of mucocutaneous autoimmune diseases, viral diseases, diseases with immune modulated mechanisms, and genetically inherited diseases. The diagnosis of this disease is commonly made clinically with histopathological and immunofluorescence investigations. ${ }^{1,2}$ Benign Mucous Membrane Pemphigoid (MMP) is one of the VB diseases that mostly affect the mucous membranes. MMP is one of the rare diseases of oral mucous membrane with an incidence of 2-10 cases per 100,000 population. ${ }^{3,4}$

The course of this disease is considered less severe compared to Pemphigus. In this disease, immunoglobulins, i.e., $\operatorname{IgG}, \operatorname{IgA}$, and $\operatorname{IgM}$ autoantibodies move towards the different antigens such as laminin 332, 311, targeting bullous antigen, collagen type-VII, $\alpha 6 \beta 4$-integrin, and unidentified basal membrane zone antigens in the basement membrane.5,6 MMP is mostly prevalent in elderly females. ${ }^{6}$ Different mucosal sites can be involved, such as oral mucosa, oropharynx, larynx, ocular, and genital mucosa. Sometimes, it also appears on the skin mostly involved head, neck, and upper torso. ${ }^{1,3}$ The typical characteristic sign of MMP is the mucosal erosion, blister formation, and followed by scarring. $1^{6}$ Other intra-oral clinical features include vesicles, desquamative gingivitis, erosions covered by pseudomembranous slough, and ulcers. ${ }^{7}$

\section{CASE PRESENTATION}

A 48-year-old female patient reported to the outpatient department of Oral and Maxillofacial Pathology of Modern Dental College, Indore, India, with a chief complaint of ulcers in the mouth for eight months (Figure 1). She noticed the appearance of multiple ulcers on the right side of buccal mucosa that healed and reappeared after 10 to 15 days, mostly at the same site, preceded by boils that burst in 3-4 days. History of itching on the scalp was also present. The patient had no history of deleterious oral habits. No recent drug history was reported by the patient. She had no relevant family history.

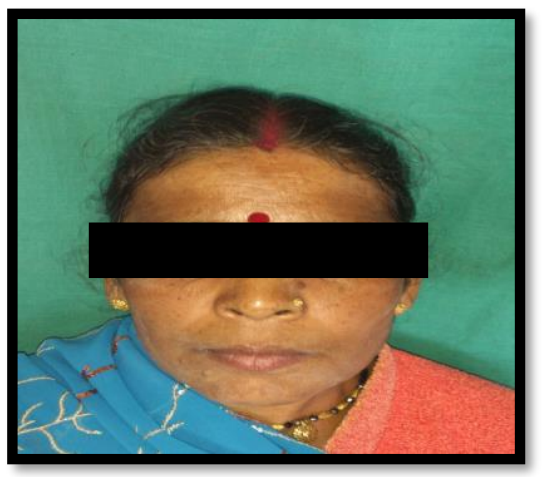

Figure 1. Clinical Appearance of the Patient

General Examination revealed that the patient was moderately built, cooperative. Vital signs were within the normal range, no sign of pallor in lower palpebral conjunctiva and nails, no sign of edema and cyanosis 
reported. The examination of lymph nodes showed bilateral submandibular lymphadenopathy. Extraoral examination showed that lips were competent, no abnormality was detected in TMJ's, no sign of rash or erythema on the skin, and no gross facial asymmetry. Areas of excoriation were present on the scalp.

Intraoral soft tissue examination revealed a grayishwhite lesion on the right buccal mucosa with diffuse borders. The lesion was extending anteroposteriorly from the commissure of lips to the retromolar area, superior-inferiorly in the upper and lower buccal vestibule, and interspersed with minute pinpoint ulcers of 0.5 to $2 \mathrm{~mm}$ in size (Figure 2). Localized desquamation of marginal gingiva was present concerning 45, 46, 47 teeth (Figure 3). The entire lesion was scrapable. However, tissue tags remained with bleeding and tender. Intraoral hard tissue examination showed that patient had missing teeth, i.e., $16,17,26$, a carious tooth with 46 , occlusal facets on $36,37,46$ teeth and generalized calculus and gingival inflammation. Based on the above-mentioned clinical findings, a provisional diagnosis of Erosive lichen planus and differential diagnosis of Vesiculobulouos Lichen planus, Pemphigus, and Pemphigoid were given.

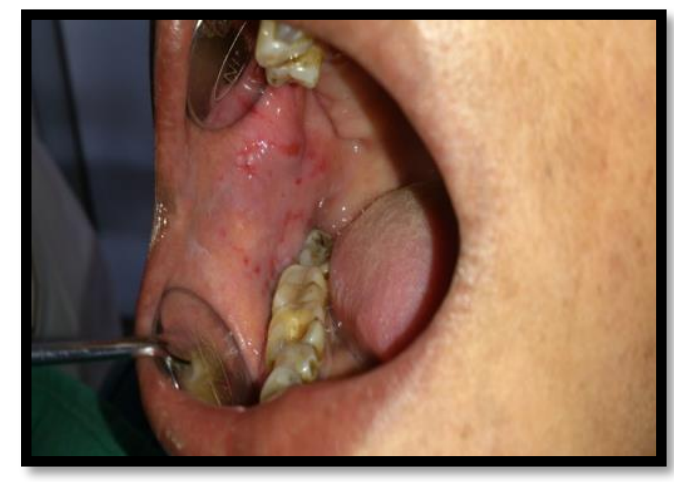

Figure 2. Clinical Examination of the Lesion

The patient had undergone haematological and urine examination. Blood examination revealed average values for haemoglobin, haematocrit, bleeding, and clotting time, and blood sugar level. The urine analysis report was also found negative. An incisional biopsy specimen was obtained. The specimen was firm in consistency, irregular in shape, and $1.1 \times 0.3 \times 0.2 \mathrm{~cm}$ in size and examined for histopathological features (Figure 4). Histopathology highlighted parakeratinized stratified squamous epithelium of different thicknesses with subepithelial split and degeneration of basal cells



Figure 3. Presence of Localized

Desquamation of Marginal Gingiva with respect to $45,46,47$

in some areas. Inflammatory cell infiltrates, eosinophils, and vascularity seen in connective tissue stroma (Figure 5). A final definitive diagnosis of benign mucous membrane pemphigoid was given based on the clinical findings and investigations. The patient was prescribed triamcinolone acetonide steroid ointment for topical application three times a day and recalled after one month. Improvement was observed after four weeks of steroid application, and then the patient was asked to taper the dose.

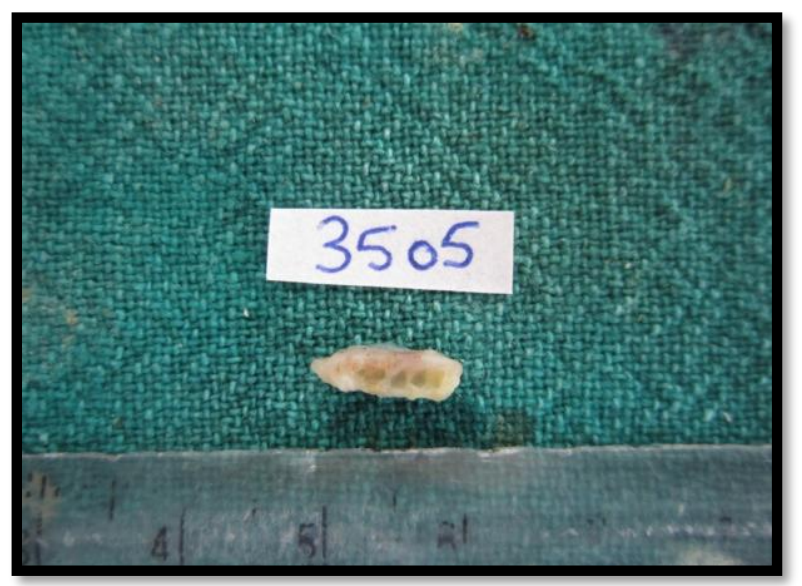

Figure 4. Specimen Obtained for Incisional Biopsy

\section{DISCUSSION}

Benign mucous membrane pemphigoid is a group of chronic blistering, mucocutaneous autoimmune diseases in which autoantibodies move against one or more components of the basement membrane. The first case of MMP was reported in 1794 by Wickmann. 8 The exact etiology of MMP is still not clear. There are many possible etiological risk factors such as severe 


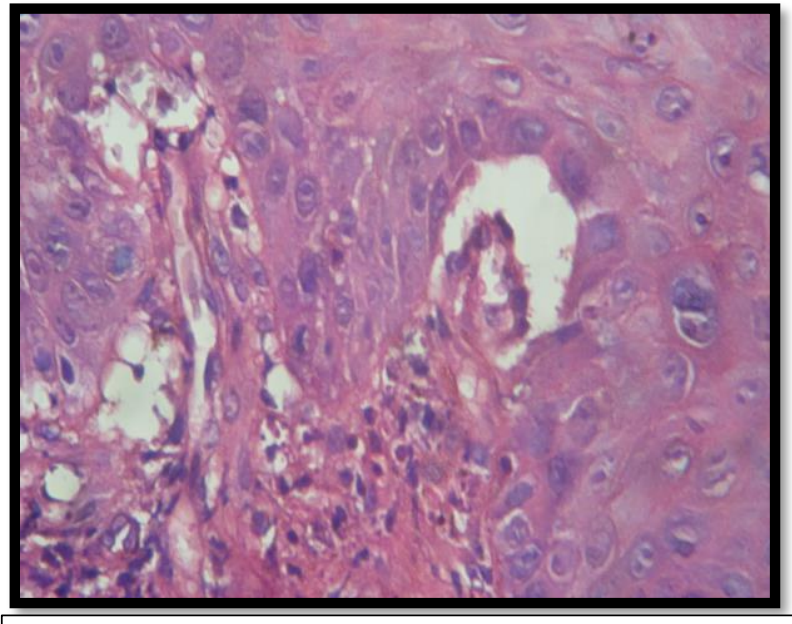

Figure 5. Histopathological Examination of the Lesion

inflammatory injury of the mucosa, drugs (Dpenicillamine, indomethacin, clonidine), virus, ultraviolet rays, and genetic susceptibility (biomarker HLA DQB1*03:01) have been reported in the existing literature.9 MMP is an autoimmune, VB lesion in which cytokines and leukocyte enzymes release through complement system by sequestration of neutrophil and followed by cell lysis possibly from complement system, results in loss of adhesion of basal cell-basement membrane leading to the formation of a vesicle under the epithelium.1o Body induced a specific protein such as $168-\mathrm{KDa}, \alpha 6$ integrin which is found exclusively in oral mucous membrane pemphigoid."

MMP occurs mostly between 40 and 50 years, and females are affected more frequently than males by a $2: 1$ ratio. Oral lesions are the typical characteristic feature seen in most of the patients. It affects mostly oral mucosa $(85 \%)$, and other mucosae such as nasal mucosa, oropharynx, conjunctiva, and genital mucosa. ${ }^{12-15}$ Oral lesions of pemphigoid begin as either vesicles or bullae. The oral blisters rupture, leaving large, superficial, ulcerated, and denuded areas of mucosa. The ulcerated lesions are usually painful and persist for weeks to months if untreated. Gingival involvement produces a clinical reaction pattern termed desquamative gingivitis. ${ }^{16}$ The lesion healed with a scar and followed by hyperpigmentation. ${ }^{15}$ In some cases, complications such as hoarseness of voice, ocular defect, and airway obstruction may occur. ${ }^{12,15}$ Skin lesions are not much common and mostly found on the face, neck, scalp, abdomen, and limbs. ${ }^{17}$

The biopsy of the lesion shows a split between the surface epithelium and the underlying connective tissue. A mild chronic inflammatory cell infiltrate is present in the superficial submucosa. Direct immunofluorescence studies show a continuous linear band of immunoreactions at the basement membrane zone. The immune deposits consist primarily of IgG and $\mathrm{C}_{3}$, although IgA and IgM may also be identified. ${ }^{18}$ The topical application of steroids on the lesion is advised. If topical steroids are unsuccessful, systemic steroids plus other immunosuppressive agents can be prescribed.

\section{CONCLUSION:}

Based on the different clinical evidence and histopathological findings, mucous membrane pemphigoid resembles a variety of diseases. The investigations for differentiating various types of pemphigoid are specific, expensive, and not routinely used. Therefore, this entity requires a comprehensive clinical, and histopathological evaluation. Early detection and prompt treatment recommended avoiding potential complications.

\section{REFERENCES:}

1. Rastogi V, Sharma R, Misra S, Yadav L. Diagnostic procedures for autoimmune vesiculobullous diseases: A review. J Oral Maxillofac Pathol.2014;18(3):390.

2. Laskaris G. $2^{\text {nd }}$ ed. Ch 4 . Georg Thieme Verlag. Pocket atlas of oral diseases; 2006 pp. 101-6.

3. Xu HH, Werth VP, Parisi E, Sollecito TP. Mucous membrane pemphigoid. Dent Clin North Am.2013;57:611-30.

4. Dear JW, Lilitkarntakul P, Webb DJ. Are rare diseases still orphans or happily adopted? The challenges of developing and using orphan medicinal products. Br J Clin Pharmacol.2006;62:264-71.

5. Bruch-Gerharz D, Hertl M, Ruzicka T. Mucous membrane pemphigoid: Clinical aspects, immunopathological features and therapy. Eur J Dermatol.2007;17:191-200.

6. Schmidt E, Zillikens D. Pemphigoid diseases. Lancet.2013;381:320-32.

7. Scully C, Carrozzo M, Gandolfo S, Puiatti P, Monteil R. Update on mucous membrane pemphigoid: A heterogeneous immune-mediated subepithelial blistering entity. Oral Surg Oral Med Oral Pathol Oral Radiol Endod.1999;88:56-68.

8. Wickmanns JE. Ideas on the diagnosis. Vol. 1. Hanover: Helwig;1894. p. 89.

9. Delgado JC, Turbay D, Yunis EJ, Yunis JJ, Morton ED, Bhol K, et al. A common major histocompatibility 
complex Class II allele HLA-DQB1 ${ }^{*} 0301$ is present in clinical variants of pemphigoid. Proc Natl Acad Sci U S A.1996;93:8569-71.

10. Scully C, Lo Muzio L. Oral mucosal diseases: Mucous membrane pemphigoid. $\mathrm{Br} \mathrm{J}$ Oral Maxillofac Surg.2008;46:358-66.

11. Sami N, Bhol KC, Ahmed AR. Treatment of oral pemphigoid with intravenous immunoglobulin as monotherapy. Long-term follow-up: Influence of treatment on antibody titres to human alpha6 integrin. Clin Exp Immunol.2002;129:533-40.

12. Ata-Ali F, Ata-Ali J. Pemphigus vulgaris and mucous membrane pemphigoid: update on etiopathogenesis, oral manifestations and management. J Clin Exp Dent.2011;3(3):e246-250.

13. Mostafa MI, Hassib NF, Nemat AH. Oral mucous membrane pemphigoid in a 6-year-old boy: diagnosis, treatment and 4 years follow-up. Int $\mathrm{J}$ Paediatr Dent.2010;20(1):76-79.
14. Schifter M, Yeoh SC, Coleman H, Georgiou A. Oral mucosal diseases: the inflammatory dermatoses. Aust Dent J.2010;55(Suppl 1):23-38.

15. Rajendran R, Sivapathasundharam B. Shafer's Textbook of Oral Pathology; Elsevier: 6th ed; 2009. p. 822.

16. Gagari E, Damoulis PD. Desquamative gingivitis as a manifestation of chronic mucocutaneous disease. JDDG: Journal der Deutschen Dermatologischen Gesellschaft.2010;9(3):184-7.

17. Bagan J, Lo Muzio L, Scully C. Mucosal disease series - number III: mucous membrane pemphigoid. Oral Dis.2005;11:197-218.

18. Arvind Babu RS, Chandrasekar P, Chandra KL, Reddy GS, Kumar KK, Ramana Reddy BV. Immunofluorescence and its application in dermatopathology with oral manifestations: Revisited. J Orofac Sci.2013;5:2-8. 